\title{
Recasting a biologically motivated computational model within a Fechnerian and random utility framework
}

\author{
Clintin P. Davis-Stober \\ Corresponding Author \\ Department of Psychological Sciences \\ 219 McAlester Hall \\ University of Missouri at Columbia \\ Columbia, MO 65211, USA \\ cstober2@gmail.com \\ Nicholas Brown \& Sanghyuk Park \\ Department of Psychological Sciences \\ 210 McAlester Hall \\ University of Missouri at Columbia \\ Columbia, MO 65211, USA \\ nrbrown87@gmail.com 63 sanghyuk.park@mail.missouri.edu \\ Michel Regenwetter \\ Department of Psychology \\ 603, E. Daniel Street \\ University of Illinois at Urbana-Champaign \\ Champaign, IL 61820, USA \\ regenwet@illinois.edu
}

\begin{abstract}
The selective integration model of Tsetsos et al. (2016a) is a biologically motivated computational framework that aims to model intransitive preference and choice. Tsetsos et al. (2016a) concluded that a noisy system can lead to violations of transitivity in otherwise rational agents optimizing a task. We show how their model can be interpreted from a Fechnerian perspective and within a random utility framework. Specifically, we spell out the connection between the selective integration model and two probabilistic models of transitive preference, weak stochastic transitivity and the triangle inequalities, tested by Tsetsos et al. (2016a).
\end{abstract}

Keywords: Selective integration, Fechnerian model, random utility, weak stochastic transitivity, triangle inequalities. 


\section{Introduction.}

Mathematical psychology has a long tradition of axiomatic approaches to theoretical and empirical psychology (see, e.g. Birnbaum, 2005; Birnbaum and Beeghley, 1997; Dagsvik, 2008; Davis-Stober, 2012; Davis-Stober and Brown, 2013; Falmagne, 1978; Karabatsos, 2005; Krantz et al., 1971; Luce, 1956, 1959, 1977, 2000; Luce et al. 1990; Luce and Marley, 2005; Luce and Suppes, 1965, Marley, 2002; Myung et al. 2005; Narens, 1985; Regenwetter et al., 2010, 2011; Regenwetter and Davis-Stober, 2012; Roberts, 1979; Scott and Suppes, 1958; Davis-Stober, 2009; Suppes et al., 1989. Suppes and Zanotti, 1992; Tversky, 1969, Wakker and Tversky, 1993, Yellott, 1977, for examples spanning six decades). One of the core ideas underlying the axiomatic approach is to formulate mathematically concise properties, called axioms, that differentiate entire classes of theories from each other. One goal of empirical axiom testing is to determine the viability of various classes of theories, at a maximally general level.

Arguably the most prominent axiom in mathematical psychology is transitivity of a binary relation. In decision making, transitivity of preference embodies the idea that preferences over choice alternatives admit numerical measurement according to a scale; such a measurement function is typically referred to as a utility function. Understanding the restrictiveness of an axiom is of great importance for theory development and for empirically differentiating between theories that are/are not consistent with the axiom. In the case of transitivity, as the number of choice alternatives increases, even for a small double digit number of options, the axiom is quite restrictive as the vast majority of possible binary relations violate it 11 Hence, transitivity provides researchers a strong, empirically testable property to differentiate between theories that are highly structured in one particular way and all others.

A guiding principle in axiomatizations and in axiom testing is to keep careful track of auxiliary assumptions and their role in explaining behavior. This allows scholars to eliminate unneeded assumptions while retaining crucial ones, in their effort to achieve maximal generality in psychological theory. It also guards against accidentally rejecting adequate theories because of inadequate auxiliary assumptions. While this perspective offers generality and rigor, it is less useful for explicitly modeling cognitive processes. Researchers following an axiomatic approach are primarily concerned with what behavior is ruled in or out, not so much with how that behavior is generated.

\footnotetext{
${ }^{1}$ For example, for 20 choice alternatives, there are $20 ! \approx 10^{18}$ transitive, complete, asymmetric relations (complete rankings without ties), out of $2^{180} \approx 10^{54}$ complete, asymmetric relations.
} 
Contrasting the axiomatic approach, a more modern strand of research in mathematical psychology aims to model the latent and unobservable cognitive processes that generate observed behavior and to create diagnostic experiments that allow different process models to compete in accounting for empirical data (see, e.g., Brown and Heathcote, 2005, 2008; Busemeyer and Townsend, 1993; Otter et al., 2008; Ratcliff and Rouder, 1998; Ratcliff and Starns, 2009; Roe et al., 2001; Smith and Ratcliff, 2009; Trueblood et al., 2014; Townsend et al., 2012; Tsetsos et al., 2016a; Wagenmakers et al. 2007). This perspective diverges from the axiomatic approach primarily in level of abstraction. While the axiomatic approach operates at maximum generality, researchers following such a "computational" approach tend to model the cognitive process under investigation via explicitly defined functions and related parameters. By operating at a lower level of abstraction, these models tend not to be as general. However, they can be very useful for understanding cognitive phenomena.

The axiomatic approach and the computational approach oftentimes seem to proceed in isolation. We consider a case in which they explicitly connect. Tsetsos et al. (2016a) used a dynamic computational process model to formulate general claims about intransitivity of preferences. In this article, we explore in more detail what can be gained by connecting the two approaches. We examine the computational model of Tsetsos et al. (2016a) under an axiomatic lens. We embed their model within a larger, and more general class of choice theory. In doing so, we are able to precisely relate fundamental properties of choice with different parameter values in their model.

Tsetsos et al. (2016a) presented a biologically motivated computational model, called the selective integration model (henceforth SI) stated recursively as a sequential sampling process. For each pairwise choice among two options, the model involves two accumulators that integrate samples from attributes associated with those two alternatives. These samples are subject to perceptual noise. The model then applies rank-dependent weighting to the noisy samples before adding decision noise. The core idea is that (Tsetsos et al., 2016a, p.1) "choice attributes (e.g., the expense, weather, or culture encountered on a holiday) are sampled in turn [...], and integrated toward a cumulative decision variable [...]. Under selective integration, the gain of processing on each attribute $i$ of an alternative depends on its rank within that attribute, with a selective gating parameter $w(0<w<1)$ controlling the reduction in gain for the weakest attribute value. [...] Selective integration thus makes decisions sensitive to the relative ranks of the alternatives within each attribute, over and above their cumulative average value." For certain values of choice attributes and certain parameter configurations, the SI model generates intransitive choices.

Tsetsos et al. (2016a) extensively employed computer simulations to study the 
mathematical properties of their model, to evaluate its statistical fit to data, and to estimate its parameters. To provide support for the SI model, Tsetsos et al. (2016a) carried out a series of experiments to test the transitivity axiom. Using contemporary methods from the axiom testing literature (Regenwetter et al., 2010, 2011, 2014), they reported that 11 of their 21 participants violated the axiom of transitivity ${ }^{2}$, Combining this finding with the reported statistical performance of their own model on several data sets, they concluded that "intransitivity can be provoked in most individuals" in the fashion predicted by their model and to support their general claim that "[e]conomic irrationality is optimal during noisy decision making." In a commentary that focussed exclusively on Tsetsos et al.'s data (not their model), Davis-Stober et al. (2016) suggested that their reported violations of transitivity are aggregation artifacts caused by a particular experimental design and by questionable data pooling across experimental conditions. In other words, Davis-Stober et al. (2016) called into question whether participants in the Tsetsos et al. study actually displayed economic irrationality. More specifically, Davis-Stober et al. reported that only 1 of 21 participants violated a classical property from the axiom testing literature called "weak stochastic transitivity," (at a 5\% significance level) consistently across experimental conditions - see Tsetsos et al. (2016b) for a rejoinder. Here, we ignore all empirical questions about Tsetsos et al.'s data. We also ignore all questions about the interpretability of their empirical findings. Instead, we focus on mathematical structure and what it means.

We investigate the relationships between the selective integration model and classic random utility and Fechnerian theory. This analysis provides an explicit explanation for when (and why) the SI model can violate well-known choice properties. Specifically, we show that when gating is not present $(w=1)$, the SI model becomes a weak utility model and a unidimensional and noncoincident random utility model. By being a member of these classes, the SI model without gating must satisfy both weak stochastic transitivity and the triangle inequalities. We also demonstrate that when gating is not present, the SI model reduces to a two-parameter model, with perceptual and decision noise being theoretically conflated. When selective gating is present, we demonstrate that the SI model can be interpreted as (or approximated by) a context-dependent random utility model, as well as a Fechnerian model, and need not satisfy either weak stochastic transitivity or the triangle inequalities.

\footnotetext{
${ }^{2}$ One of the 11 violations of "weak stochastic transitivity" was incompatible with their model.
} 


\section{The selective integration model.}

Throughout, let $\mathcal{S}$ denote a finite set of stimuli (Tsetsos et al. concentrate on $|\mathcal{S}|=3$.) Our notation by and large follows that of Tsetsos et al. $(2016 \mathrm{a}) \mathrm{I}^{3}$ The following definition specifies the model predictions for a binary forced choice between two options, $A$ and $B$. Throughout, we use boldfaced symbols to denote random variables.

Definition. Let $t$ denote the current discrete time step $4^{4}$ Let $s_{A}(t), s_{B}(t)$ denote stimulus intensities (e.g., bar heights in pixels) at $t$, and let $\boldsymbol{\rho}_{A}(t), \boldsymbol{\rho}_{B}(t)$ be standard normal random variables independent of each other and independent of $t$. Let $\boldsymbol{X}_{A}(t), \boldsymbol{X}_{B}(t)$ denote the corrupted subjective representations of the stimuli, due to (early) noise with noise parameter ${ }^{5} \sigma$, defined by

$$
\boldsymbol{X}_{A}(t)=\sigma \cdot \boldsymbol{\rho}_{A}(t)+s_{A}(t), \quad \boldsymbol{X}_{B}(t)=\sigma \cdot \boldsymbol{\rho}_{B}(t)+s_{B}(t) .
$$

Let $w \in[0,1]$ be a selective gating parameter ${ }^{6}$ and define a gain function $\theta$ as the step-function 7

$$
\theta(x)= \begin{cases}1, & \text { if } x>0 \\ w & \text { if } x<0\end{cases}
$$

Define

$$
\boldsymbol{I}_{A}(t)=\theta\left(\boldsymbol{X}_{A}(t)-\boldsymbol{X}_{B}(t)\right) \cdot \boldsymbol{X}_{A}(t), \quad \boldsymbol{I}_{B}(t)=\theta\left(\boldsymbol{X}_{B}(t)-\boldsymbol{X}_{A}(t)\right) \cdot \boldsymbol{X}_{B}(t),
$$

to serve as momentary inputs to accumulators $\boldsymbol{Y}_{A}(t)$ and $\boldsymbol{Y}_{B}(t)$ that integrate the

\footnotetext{
${ }^{3}$ We deviate primarily in cases where the original notation creates ambiguity.

${ }^{4}$ Tsetsos et al. (2016a) identify $t$ with the currently displayed choice dimension/attribute. Consistent with Tsetsos et al., we do not treat $t$ as a random variable.

${ }^{5}$ We assume $0 \leq \sigma \in \mathbb{R}$, without upper bound.

${ }^{6}$ The original paper is inconsistent in whether $w \in(0,1)$ or $w \in[0,1]$, but effectively uses $[0,1]$.

7 Tsetsos et al. (2016a) left $\theta(0)$ undefined. This only introduces ambiguity for the case $\sigma=0$. For this case, they suggest that the gain function is applied probabilistically, i.e., gating happens with probability .5 (personal communication). Tsetsos et al. (2016a) used $w$ inconsistently across the main manuscript, appendix, supplementary materials, and computer code: For example, $w$ in their Figure 1 corresponds to $1-w$ in their mathematical model statement. We use $w$ as defined in Equation 3 of their main document.
} 
attribute values of the two sequences $A$ and $B$ according to the difference equations

$$
\begin{aligned}
& \boldsymbol{Y}_{A}(t)=(1-\lambda) \cdot \boldsymbol{Y}_{A}(t-1)+\boldsymbol{I}_{A}(t)+\xi \cdot \boldsymbol{\zeta}_{A}(t), \\
& \boldsymbol{Y}_{B}(t)=(1-\lambda) \cdot \boldsymbol{Y}_{B}(t-1)+\boldsymbol{I}_{B}(t)+\xi \cdot \boldsymbol{\zeta}_{B}(t),
\end{aligned}
$$

with $\lambda$ an integration leak 8 late noise 9 and $\boldsymbol{\zeta}_{A}(t), \boldsymbol{\zeta}_{B}(t)$ two standard normal random variables independent of each other and of $t$. The accumulators are initialized as

$$
\boldsymbol{Y}_{A}(0)=\boldsymbol{Y}_{B}(0)=0 .
$$

At the end of the presentation of stimuli (e.g., $\mathrm{t}=9$, in Tsetsos et al's Experiment 1), the model chooses sequence A if $\boldsymbol{Y}_{A}(t)>\boldsymbol{Y}_{B}(t)$, chooses sequence B if $\boldsymbol{Y}_{B}(t)>\boldsymbol{Y}_{A}(t)$, and chooses either with equal probability if $\boldsymbol{Y}_{A}(t)=\boldsymbol{Y}_{B}(t)$.

\section{Classical choice models and a recent extension.}

We now provide a definition of various classical choice models grounded in the axiomatic literature (see, e.g., Block and Marschak, 1960 Hey and Orme, 1994; Loomes and Sugden, 1995 Luce, 1959; Luce and Suppes, 1965; McFadden, 1973; Thurstone, 1927; Yellott, 1977).

Definition. According to a Fechnerian model, the binary choice probability $P_{A B}$ of choosing $A$ over $B$ is of the form

$$
P_{A B}=F\left(s_{A B}\right) \quad(\forall \text { distinct } A, B \in \mathcal{S}),
$$

with $F$ a cumulative distribution function (CDF), $F(0)=\frac{1}{2}$, and with $s_{A B} \in \mathbb{R}$ the strength of preference in favor of $A$ over $B$ (i.e., $s_{A B}>0$ when $A$ is preferred to $B$ ). A logistic CDF yields the logit model, whereas a normal CDF yields the probit model. Standard logit and probit models use the strength of preference function

$$
s_{A B}=s_{A}-s_{B},
$$

with $s_{A}$ and $s_{B}$ subjective values of the options $A$ and $B$, respectively. However, in general, Fechnerian models, including logit and probit models, can use any strength

${ }^{8}$ Tsetsos et al. (2016a) did not specify the range of $\lambda$. They expect that $\lambda \in[0,1)$; most likely in the range $[0,0.5]$ (personal communication).

${ }^{9}$ We assume $0 \leq \xi \in \mathbb{R}$, without upper bound. 
of preference function $s_{A B}$ satisfying

$$
s_{A B}=-s_{B A} \quad(\forall A, B \in \mathcal{S}) .
$$

For example, some theories, such as regret theory (Loomes and Sugden, 1982), provide a strength of preference directly among pairs of options, without subjective values attached to any individual options. In such cases, even though Fechnerian models are well defined, they cannot be of a form involving a strength of preference function as in Equation 1. Note also that theories without numerical strengths of preferences, such as many non-numeric heuristic models, do not allow a Fechnerian specification.

According to a weak utility model, there exists a real-valued function $u$ on the set of stimuli such that

$$
P_{A B} \geq \frac{1}{2} \Leftrightarrow u(A) \geq u(B) \quad(\forall \text { distinct } A, B \in \mathcal{S}) .
$$

According to a unidimensional and noncoincident random utility model, there exist jointly distributed random variables $\left(\boldsymbol{U}_{S}\right)_{S \in \mathcal{S}}$ and a probability measure $\operatorname{Pr}$ [with $\operatorname{Pr}\left(\boldsymbol{U}_{S}=\boldsymbol{U}_{S^{\prime}}\right)=0$ when $S \neq S^{\prime}$ ] such that

$$
P_{A B}=\operatorname{Pr}\left(\boldsymbol{U}_{A}>\boldsymbol{U}_{B}\right) \quad(\forall \text { distinct } A, B \in \mathcal{S}) .
$$

Random utility models with joint extreme value, respectively joint normal distributions, form logit and probit models, respectively.

Let $\Pi_{\mathcal{S}}$ denote the collection of all linear orders (rankings without ties) of elements of $\mathcal{S}$ and for $\pi \in \Pi, A \in \mathcal{S}, B \in \mathcal{S}, A \neq B$, let $A \pi B$ denote that $\pi$ ranks $A$ ahead of $B$ (e.g., $A$ is 'preferred' to $B$ in 'preference state' $\pi$ ). According to the linear ordering (random preference) model, there exists a probability distribution assigning probability $p_{\pi}$ to each $\pi \in \Pi$ such that

$$
P_{A B}=\sum_{\substack{\pi \in \Pi \\ A \pi B}} p_{\pi} \quad(\forall \text { distinct } A, B \in \mathcal{S}) .
$$

Next, we summarize some well-established results about these classical models (see, e.g., Block and Marschak, 1960, Cohen and Falmagne, 1978; Luce and Suppes, 1965; Yellott, 1977).

LEMMA 1.

- Every Fechnerian model with a strength of preference function $s_{A B}$ as in Equa- 
tion 1 is a weak utility model. Hence, standard probit and logit models are weak utility models.

- Every weak utility model implies weak stochastic transitivity, namely

$P_{A B} \geq \frac{1}{2} \quad$ AND $\quad P_{B C} \geq \frac{1}{2} \quad \Rightarrow \quad P_{A C} \geq \frac{1}{2} \quad(\forall$ distinct $A, B, C \in \mathcal{S})$.

Conversely, when weak stochastic transitivity holds, then there exists a weak utility model (not necessarily logit or probit) of the binary choice probabilities.

- Binary choice probabilities satisfy a unidimensional and noncoincident random utility model if and only if they satisfy a linear order (random preference) model. Such binary choice probabilities imply the triangle inequalities, namely

$$
P_{A B}+P_{B C}-P_{A C} \leq 1 \quad(\forall \text { distinct } A, B, C \in \mathcal{S}) .
$$

For $|\mathcal{S}| \leq 5$ the triangle inequalities are necessary and sufficient conditions, on a set of binary choice probabilities, for the existence of a unidimensional and noncoincident random utility model, and an equivalent linear order (random preference) model. For $|\mathcal{S}|>5$ they are only necessary, but not sufficient.

- Binary choice probabilities that satisfy the weak utility model need not satisfy a unidimensional and noncoincident random utility model, and hence need not satisfy a linear order (random preference) model.

- Binary choice probabilities that satisfy a unidimensional and noncoincident random utility model, and hence satisfy a linear order (random preference) model, need not satisfy a weak utility model.

- Neither weak stochastic transitivity nor the triangle inequalities imply each other.

- Classical logit and probit models jointly satisfy i) Fechnerian, ii) weak utility, iii) unidimensional and noncoincident random utility, and iv) linear order (random preference) models. In particular, they jointly satisfy weak stochastic transitivity and the triangle inequalities.

- A random utility model need not be a Fechnerian model.

Weak stochastic transitivity and the triangle inequalities are the two most prominent probabilistic models to represent choices induced by transitive preferences (see 
also Regenwetter et al., 2010, 2011, for a detailed discussion). Tsetsos et al. (2016a) reported that selective integration can violate weak stochastic transitivity. They reported statistical tests of weak stochastic transitivity to provide indirect evidence of intransitive preference consistent with selective integration. They also statistically tested one of the triangle inequalities but did not specify how this test relates to their theory other than to provide an alternative test of transitive preferences.

Last, we introduce a recent extension to classical random utility models (Regenwetter and Müller-Trede, 2016). Let $\Gamma$ denote a finite set of contexts. We write $P_{A B}^{\gamma}$ for the binary choice probability of choosing $A$ over $B$ in context $\gamma \in \Gamma$.

Definition. Let $\left(\boldsymbol{U}_{A}^{\gamma}\right)_{A \in \mathcal{S}, \gamma \in \Gamma}$ be a family of jointly distributed real-valued random variables. For any context $\gamma \in \Gamma$, we say that $\left(\boldsymbol{U}_{A}^{\gamma}\right)_{A \in \mathcal{S}}$ is (marginally) noncoincident if, writing $\operatorname{Pr}$ for the probability measure governing their joint distribution, it holds that for any distinct $A, B \in \mathcal{S}, \operatorname{Pr}\left(\boldsymbol{U}_{A}^{\gamma}=\boldsymbol{U}_{B}^{\gamma}\right)=0$. A context-dependent family of binary choice probabilities $P_{A B}^{\gamma}, A, B \in \mathcal{S}$, with $A \neq B$ and $\gamma \in \Gamma$ satisfies a contextdependent (noncoincident) random utility model if there exists a family of jointly distributed random variables, $\left(\boldsymbol{U}_{A}^{\gamma}\right)_{A \in \mathcal{S}, \gamma \in \Gamma}$, with each $\left(\boldsymbol{U}_{A}^{\gamma}\right)_{A \in \mathcal{S}}$ noncoincident, such that

$$
P_{A B}^{\gamma}=\operatorname{Pr}\left(\boldsymbol{U}_{A}^{\gamma}>\boldsymbol{U}_{B}^{\gamma}\right) \quad(A, B \in \mathcal{S}, A \neq B) .
$$

As Regenwetter and Müller-Trede (2016) discuss in detail, context-dependent random utility models may or may not imply the triangle inequalities, depending on the definition of context and on the joint distribution of the random variables. For an empirical example see Müller-Trede et al. (2015).

\section{Properties of the selective integration model.}

In this section, we provide closed form, non-recursive solutions to two special cases of SI, demonstrating that SI can be interpreted as both a Fechnerian model and a (context-dependent) random utility model. We then develop a closed form, non-recursive approximation to the unrestricted SI model (i.e., the SI model under no parametric constraints). We observe that our approximation is both a Fechnerian model and a (context-dependent) random utility model.

In Section 4.1, we consider the SI model when $w=1$. This is the "lossless" version of SI, without selective gating. This version of SI is used by Tsetsos et al. (2016a) primarily as a reference model when examining the impact of selective gating on choice. In Section 4.2, we consider a three parameter variant of SI in which $\sigma=0$ (early noise is omitted) but all other parameters are free to vary over their respective 
ranges. Tsetsos et al. (2016a) reported that this version of SI outperformed the full SI model, and all other variants, via a BIC model comparison, in each of their four experiments. In Section 4.3, we develop our approximation to the unrestricted SI model and examine its properties.

\subsection{Selective integration model when $w=1$.}

We now show that if $w=1$ then SI can be re-written as: a Fechnerian model, a probit model with a specialized strength of preference function, a weak utility model, and a unidimensional and noncoincident random utility model, hence an equivalent random preference model. Therefore, it must satisfy both weak stochastic transitivity and the triangle inequalities.

Lemma 2. Let $w=1$ and let $A \in \mathcal{S}$ be any choice alternative. Then the accumulator value for $A$ at time $t$ is as follows,

$$
\boldsymbol{Y}_{A}(t)=v_{A}+\boldsymbol{\epsilon}
$$

where

$$
v_{A}=\sum_{j=1}^{t}(1-\lambda)^{t-j} s_{A}(j)
$$

and

$$
\boldsymbol{\epsilon} \sim N\left(0, \frac{(1-\lambda)^{2 t}-1}{\lambda(\lambda-2)}\left(\sigma^{2}+\xi^{2}\right)\right)
$$

where $N\left(\psi, v^{2}\right)$ denotes a normally distributed random variable with mean $\psi$ and variance $v^{2}$. The probability of choosing $A$ over $B$ at time $t$ equals

$$
P_{A B}=\Phi\left(\frac{v_{A}}{\sqrt{\frac{2(1-\lambda)^{2 t}-2}{\lambda(\lambda-2)}\left(\sigma^{2}+\xi^{2}\right)}}-\frac{v_{B}}{\sqrt{\frac{2(1-\lambda)^{2 t}-2}{\lambda(\lambda-2)}\left(\sigma^{2}+\xi^{2}\right)}}\right)
$$

where $\Phi(\cdot)$ is the CDF for a standard normal distribution.

Let $q_{A}=\frac{v_{A}}{\sqrt{\frac{2(1-\lambda)^{2 t}-2}{\lambda(\lambda-2)}\left(\sigma^{2}+\xi^{2}\right)}}$ and $q_{B}=\frac{v_{B}}{\sqrt{\frac{2(1-\lambda)^{2 t}-2}{\lambda(\lambda-2)}\left(\sigma^{2}+\xi^{2}\right)}}$. If we define $s_{A B}=q_{A}-q_{B}$ then the Fechnerian model in Equation 6 reduces to a probit model with strength of preference function $s_{A B}$. We can also interpret $\boldsymbol{Y}_{A}(t)$ and $\boldsymbol{Y}_{B}(t)$ as random utilities such that $P_{A B}=\operatorname{Pr}\left(\boldsymbol{Y}_{A}(t)>\boldsymbol{Y}_{B}(t)\right)$, thus conforming to the definition of a unidimensional and noncoincident random utility model. By Lemma 1, if $w=1$ then SI must jointly satisfy weak stochastic transitivity and the triangle inequalities. 
When $w=1$, the SI model reduces to a two-parameter model. By examining Equation (6), it is clear that early and late noise cannot be teased apart - only their sum contributes to the model. Hence, without loss of generality, $\sigma$ and $\xi$ could be replaced with a single parameter. This is important as differentiating between early and late noise plays an important role in the interpretation of the SI model.

Proof of Lemma 2.

Let $A$ be any choice alternative from $\mathcal{S}$. From the definition of SI,

$$
\boldsymbol{X}_{A}(t) \sim N\left(s_{A}(t), \sigma^{2}\right)
$$

Under $w=1$,

$$
\boldsymbol{I}_{A}(t)=\boldsymbol{X}_{A}(t)
$$

Next, we consider the accumulator for $A$, obtaining

$$
\boldsymbol{Y}_{A}(t)=(1-\lambda) \cdot \boldsymbol{Y}_{A}(t-1)+\boldsymbol{I}_{A}(t)+\xi \cdot \boldsymbol{\zeta}_{A}(t) .
$$

By independence of $\boldsymbol{\zeta}_{A}(t)$ and $\boldsymbol{\rho}_{A}(t)$, we can rewrite the accumulator for $A$ as

$$
\boldsymbol{Y}_{A}(t)=(1-\lambda) \cdot \boldsymbol{Y}_{A}(t-1)+\boldsymbol{Z}_{A}(t)
$$

where $\boldsymbol{Z}_{A}(t) \sim N\left(s_{A}(t), \sigma^{2}+\xi^{2}\right)$. From the definition of SI, the accumulator for $A$ at time $t$ is

$$
\boldsymbol{Y}_{A}(t)=\sum_{j=1}^{t}(1-\lambda)^{t-j} \boldsymbol{Z}_{A}(j)
$$

Since $\boldsymbol{\zeta}_{A}(t)$ and $\boldsymbol{\rho}_{A}(t)$ are independent of one another and across $t, \boldsymbol{Y}_{A}(t)$ will also be a normally distributed random variable. Applying basic operations on independent normally distributed random variables yields

$$
\boldsymbol{Y}_{A}(t) \sim N\left(v_{A}, \frac{(1-\lambda)^{2 t}-1}{\lambda(\lambda-2)}\left(\sigma^{2}+\xi^{2}\right)\right),
$$

where $v_{A}=\sum_{j=1}^{t}(1-\lambda)^{t-j} s_{A}(j)$. Finally, $P_{A B}$ is obtained by observing that $\boldsymbol{D}=\boldsymbol{Y}_{A}(t)-\boldsymbol{Y}_{B}(t)$ is normally distributed with mean $v_{A}-v_{B}$ and variance $\frac{2(1-\lambda)^{2 t}-2}{\lambda(\lambda-2)}\left(\sigma^{2}+\xi^{2}\right)$. This completes the proof. 
4.2. Selective integration model when $w<1$ and $\sigma=0$.

Let

$$
\mathcal{I}_{\text {statement }}=\left\{\begin{array}{l}
1 \text { when statement is true } \\
0 \text { when statement is false. }
\end{array}\right.
$$

Let $A$ be any choice alternative and assume that $A$ is being compared to $B$. Then the accumulator $\boldsymbol{Y}_{A}(t)$ can be rewritten as a context-dependent random utility model with $\Gamma=\{A B, B C, A C\}$, i.e., the context is defined by the pair of choice alternatives being considered.

Lemma 3. Let $A$ and $B$ be any distinct pair of choice alternatives. Let $\gamma=A B$. Then the accumulator value for $A$ is of the form

$$
\boldsymbol{Y}_{A}^{\gamma}(t)=v_{A}^{\gamma}+\boldsymbol{\epsilon},
$$

where $v_{A}^{\gamma}=f_{A}^{\gamma}+w \cdot g_{A}^{\gamma}$, where $f_{A}^{\gamma}$ (resp. $g_{A}^{\gamma}$ ) is a weighted sum of attributes that favor (resp. do not favor) alternative A compared to $B$, defined as

$$
\begin{aligned}
& f_{A}^{\gamma}=\sum_{j=1}^{t}(1-\lambda)^{t-j} \mathcal{I}_{s_{A}(j)>s_{B}(j)} s_{A}(j), \\
& g_{A}^{\gamma}=\sum_{j=1}^{t}(1-\lambda)^{t-j} \mathcal{I}_{s_{A}(j)<s_{B}(j)} s_{A}(j) .
\end{aligned}
$$

The probabilistic error component, $\boldsymbol{\epsilon}$, is a normally distributed random variable,

$$
\boldsymbol{\epsilon} \sim N\left(0, \frac{(1-\lambda)^{2 t}-1}{\lambda(\lambda-2)} \xi^{2}\right) .
$$

The probability of choosing $A$ over $B$ at time $t$ equals

$$
P_{A B}=\Phi\left(\frac{v_{A}^{\gamma}}{\sqrt{\frac{2(1-\lambda)^{2 t}-2}{\lambda(\lambda-2)}} \xi^{2}}-\frac{v_{B}^{\gamma}}{\sqrt{\frac{2(1-\lambda)^{2 t}-2}{\lambda(\lambda-2)}} \xi^{2}}\right) .
$$

The proof is omitted since it is nearly identical to that of Lemma 2 .

By Equation 11 this version of SI is a Fechnerian model with a strength of pref- 
erence function that is not of the form $s_{A B}=s_{A}-s_{B}$. Rather, the two numerators on the right each depend on both $A$ and $B$, and therefore, this version of SI can permit intransitive preference, depending on the choice alternatives. Equations $7+11$ also connect this version of SI to the context-dependent random utility formulation of Regenwetter and Müller-Trede (2016): one can interpret $\boldsymbol{Y}_{A}^{\gamma}(t)$ and $\boldsymbol{Y}_{B}^{\gamma}(t)$ as context-dependent random utilities such that $P_{A B}=\operatorname{Pr}\left(\boldsymbol{Y}_{A}^{\gamma}(t)>\boldsymbol{Y}_{B}^{\gamma}(t)\right)$ for $\gamma=A B$, thus conforming to Equation 2. Hence, if $w<1$ and $\sigma=0$ then SI is a Fechnerian model and a context-dependent random utility model that, depending on the choice alternatives, may or may not satisfy weak stochastic transitivity, and may or may not satisfy the triangle inequalities.

It is worth noting that, even if $\sigma=0$ the choice stimuli can nonetheless vary. Lemma 3 would apply to the case where choice attributes are deterministic but variable, i.e., where the stimulus inputs are a known sequence controlled by the experimenter.

\subsection{An approximation to the selective integration model.}

We now demonstrate that the SI model with no parameter restrictions can be well approximated by a Fechnerian representation. When early noise and selective gating are present, the random variables $\boldsymbol{I}_{A}(t)$ and $\boldsymbol{I}_{B}(t)$ are no longer normally distributed, thus the accumulator random variables, $\boldsymbol{Y}_{A}(t)$ and $\boldsymbol{Y}_{B}(t)$, are no longer normally distributed. This makes finding a closed-form expression for the unrestricted SI model quite difficult. To this end, we construct a model to approximate SI, termed $F$-SI, which uses normal distributions to approximate the random variables, $\boldsymbol{Y}_{A}(t)$ and $\boldsymbol{Y}_{B}(t)$, leaving their expected values and variances identical to those from the unrestricted SI model. We show that, consistent with Lemma 3, F-SI is both a Fechnerian and context-dependent random utility model. We find that as late noise becomes large, and/or the selective gating parameter $w$ becomes arbitrarily close to 1, F-SI converges to the SI model. In order to define our approximation, we require the following two results.

LEMmA 4. The expected values of $\boldsymbol{I}_{A}(t)$ and $\boldsymbol{I}_{B}(t)$, denoted $E\left[\boldsymbol{I}_{A}(t)\right]$ and $E\left[\boldsymbol{I}_{B}(t)\right]$, are equal to

$$
\begin{aligned}
& E\left[\boldsymbol{I}_{A}(t)\right]=\int_{-\infty}^{\infty} \int_{-\infty}^{x_{A}} x_{A} h\left(x_{A}, x_{B}\right) d x_{B} d x_{A}+w \int_{-\infty}^{\infty} \int_{x_{A}}^{\infty} x_{A} h\left(x_{A}, x_{B}\right) d x_{B} d x_{A} \\
& E\left[\boldsymbol{I}_{B}(t)\right]=\int_{-\infty}^{\infty} \int_{-\infty}^{x_{B}} x_{B} h\left(x_{A}, x_{B}\right) d x_{A} d x_{B}+w \int_{-\infty}^{\infty} \int_{x_{B}}^{\infty} x_{B} h\left(x_{A}, x_{B}\right) d x_{A} d x_{B}
\end{aligned}
$$


where $h\left(x_{A}, x_{B}\right)$ is the probability density function for a bivariate normal distribution with mean vector, $\boldsymbol{\mu}=\left(s_{A}(t), s_{B}(t)\right)$, and covariance matrix, $\boldsymbol{\Sigma}=\left[\begin{array}{cc}\sigma^{2} & 0 \\ 0 & \sigma^{2}\end{array}\right]$.

See the Appendix for a proof.

LEMmA 5. The variances of $\boldsymbol{I}_{A}(t)$ and $\boldsymbol{I}_{B}(t)$, denoted $\operatorname{Var}\left[\boldsymbol{I}_{A}(t)\right]$ and $\operatorname{Var}\left[\boldsymbol{I}_{B}(t)\right]$, are equal to

$$
\begin{aligned}
\operatorname{Var}\left[\boldsymbol{I}_{A}(t)\right] & =\int_{-\infty}^{\infty} \int_{-\infty}^{x_{A}} x_{A}^{2} h\left(x_{A}, x_{B}\right) d x_{B} d x_{A}-\frac{\left(\int_{-\infty}^{\infty} \int_{-\infty}^{x_{A}} x_{A} h\left(x_{A}, x_{B}\right) d x_{B} d x_{A}\right)^{2}}{\phi\left(\frac{s_{A}(t)-s_{B}(t)}{\sqrt{2 \sigma^{2}}}\right)} \\
+ & w^{2} \int_{-\infty}^{\infty} \int_{x_{A}}^{\infty} x_{A}^{2} h\left(x_{A}, x_{B}\right) d x_{B} d x_{A}-\frac{\left(w \int_{-\infty}^{\infty} \int_{x_{A}}^{\infty} x_{A} h\left(x_{A}, x_{B}\right) d x_{B} d x_{A}\right)^{2}}{\phi\left(\frac{s_{B}(t)-s_{A}(t)}{\sqrt{2 \sigma^{2}}}\right)} \\
& +\phi\left(\frac{s_{A}(t)-s_{B}(t)}{\sqrt{2 \sigma^{2}}}\right)\left(\frac{\int_{-\infty}^{\infty} \int_{-\infty}^{x_{A}} x_{A} h\left(x_{A}, x_{B}\right) d x_{B} d x_{A}}{\phi\left(\frac{s_{A}(t)-s_{B}(t)}{\sqrt{2 \sigma^{2}}}\right)}-E\left[I_{A}(t)\right]\right)^{2} \\
& +\phi\left(\frac{s_{B}(t)-s_{A}(t)}{\sqrt{2 \sigma^{2}}}\right)\left(\frac{w \int_{-\infty}^{\infty} \int_{x_{A}}^{\infty} x_{A} h\left(x_{A}, x_{B}\right) d x_{B} d x_{A}}{\sqrt{2 \sigma^{2}}}-E\left[I_{A}(t)\right]\right)^{2}, \\
\operatorname{Var}^{2}\left[\boldsymbol{I}_{B}(t)\right] & =\int_{-\infty}^{\infty} \int_{-\infty}^{x_{B}} x_{B}^{2} h\left(x_{A}, x_{B}\right) d x_{A} d x_{B}-\frac{\left(\int_{-\infty}^{\infty} \int_{-\infty}^{x_{B}} x_{B} h\left(x_{A}, x_{B}\right) d x_{A} d x_{B}\right)^{2}}{\phi\left(\frac{s_{B}(t)-s_{A}(t)}{\sqrt{2 \sigma^{2}}}\right)} \\
+ & w^{2} \int_{-\infty}^{\infty} \int_{x_{B}}^{\infty} x_{B}^{2} h\left(x_{A}, x_{B}\right) d x_{A} d x_{B}-\frac{\left(w \int_{-\infty}^{\infty} \int_{x_{B}}^{\infty} x_{B} h\left(x_{A}, x_{B}\right) d x_{A} d x_{B}\right)^{2}}{\phi\left(\frac{s_{A}(t)-s_{B}(t)}{\sqrt{2 \sigma^{2}}}\right)} \\
& +\phi\left(\frac{s_{B}(t)-s_{A}(t)}{\sqrt{2 \sigma^{2}}}\right)\left(\frac{\int_{-\infty}^{\infty} \int_{-\infty}^{x_{B}} x_{B} h\left(x_{A}, x_{B}\right) d x_{A} d x_{B}}{\phi\left(\frac{s_{B}(t)-s_{A}(t)}{\sqrt{2 \sigma^{2}}}\right)}-E\left[I_{B}(t)\right]\right)^{2} \\
+ & \left.\sqrt{2 \sigma^{2}}\right) \\
+ & \left.\frac{w \int_{-\infty}^{\infty} \int_{x_{B}}^{\infty} x_{B} h\left(x_{A}, x_{B}\right) d x_{A} d x_{B}}{s_{B}}-E\left[I_{B}(t)\right]\right)^{2}
\end{aligned}
$$

See the Appendix for a proof.

Definition. Let $\boldsymbol{I}_{A}^{\prime}(t) \sim N\left(E\left[\boldsymbol{I}_{A}(t)\right], \operatorname{Var}\left[\boldsymbol{I}_{A}(t)\right]\right)$ and $\boldsymbol{I}_{B}^{\prime}(t) \sim N\left(E\left[\boldsymbol{I}_{B}(t)\right], \operatorname{Var}\left[\boldsymbol{I}_{B}(t)\right]\right)$. We construct F-SI by substituting $\boldsymbol{I}_{A}^{\prime}(t)$ and $\boldsymbol{I}_{B}^{\prime}(t)$ for $\boldsymbol{I}_{A}(t)$ and $\boldsymbol{I}_{B}(t)$ in the SI defi- 
nitions of $\boldsymbol{Y}_{A}(t)$ and $\boldsymbol{Y}_{B}(t)$ from Section 2. Define $\boldsymbol{Y}_{A}^{\prime}(t)$ and $\boldsymbol{Y}_{B}^{\prime}(t)$ via the following difference equations,

$$
\begin{aligned}
& \boldsymbol{Y}_{A}^{\prime}(t)=(1-\lambda) \cdot \boldsymbol{Y}_{A}^{\prime}(t-1)+\boldsymbol{I}_{A}^{\prime}(t)+\xi \cdot \boldsymbol{\zeta}_{A}(t), \\
& \boldsymbol{Y}_{B}^{\prime}(t)=(1-\lambda) \cdot \boldsymbol{Y}_{B}^{\prime}(t-1)+\boldsymbol{I}_{B}^{\prime}(t)+\xi \cdot \boldsymbol{\zeta}_{B}(t),
\end{aligned}
$$

where $\lambda, \xi, \boldsymbol{\zeta}_{A}(t)$, and $\boldsymbol{\zeta}_{B}(t)$ are defined as in Section 2. The F-SI accumulators are initialized as $\boldsymbol{Y}_{A}^{\prime}(0)=\boldsymbol{Y}_{B}^{\prime}(0)=0$. F-SI chooses sequence $A$ if $\boldsymbol{Y}_{A}^{\prime}(t)>\boldsymbol{Y}_{B}^{\prime}(t)$, chooses sequence $B$ if $\boldsymbol{Y}_{B}^{\prime}(t)>\boldsymbol{Y}_{A}^{\prime}(t)$ and chooses either with equal probability if $\boldsymbol{Y}_{A}^{\prime}(t)=\boldsymbol{Y}_{B}^{\prime}(t)$. It is straightforward to show that $E\left[\boldsymbol{Y}_{A}^{\prime}(t)\right]=E\left[\boldsymbol{Y}_{A}(t)\right]$, $E\left[\boldsymbol{Y}_{B}^{\prime}(t)\right]=E\left[\boldsymbol{Y}_{B}(t)\right], \operatorname{Var}\left[\boldsymbol{Y}_{A}^{\prime}(t)\right]=\operatorname{Var}\left[\boldsymbol{Y}_{A}(t)\right]$, and $\operatorname{Var}\left[\boldsymbol{Y}_{B}^{\prime}(t)\right]=\operatorname{Var}\left[\boldsymbol{Y}_{B}(t)\right]$.

Let $v_{A}^{\gamma}=\sum_{j=1}^{t}(1-\lambda)^{t-j} E\left[\boldsymbol{I}_{A}(j)\right]$ and $v_{B}^{\gamma}=\sum_{j=1}^{t}(1-\lambda)^{t-j} E\left[\boldsymbol{I}_{B}(j)\right]$, where $\gamma=$ $A B$. As in Lemma 3, we use $v_{A}^{\gamma}$ (resp. $v_{B}^{\gamma}$ ) to highlight that these terms are contextdependent in that the weighted sum of the expected values for choice sequence $A$ (resp. B) depends upon the choice sequence to which it is being compared. Using an identical approach as in Lemmas 2 and 3, we can solve for the probability of choosing $A$ over $B$ (as a function of $t$ ). We obtain,

$P_{A B}=\Phi\left(\frac{v_{A}^{\gamma}-v_{B}^{\gamma}}{\sqrt{\sum_{j=1}^{t}(1-\lambda)^{2(t-j)}\left(\operatorname{Var}\left[\boldsymbol{I}_{A}(j)\right]+\xi^{2}\right)+\sum_{j=1}^{t}(1-\lambda)^{2(t-j)}\left(\operatorname{Var}\left[\boldsymbol{I}_{B}(j)\right]+\xi^{2}\right)}}\right)$.

Similar to Lemma 3, F-SI is a Fechnerian model and a context-dependent random utility model that, depending on the choice alternatives, may or may not satisfy WST and may or may not satisfy the triangle inequalities.

Next, we present a simulation study examining how closely F-SI approximates the SI mode $\sqrt{10}$ Both models are functions of the same parameters: $\sigma, w, \lambda$, and $\xi$. In our simulation study, we fixed $\sigma=15$ and systematically varied the remaining three parameters. We used choice stimuli similar to the "cyclic" trials in Experiment 4 of Tsetsos et al. (2016a). We considered two choice sequences, $A$ and $B$, with $s_{A}(1)=115, s_{A}(2)=95, s_{A}(3)=75$ and $s_{B}(1)=75, s_{B}(2)=115, s_{B}(3)=95$. We varied $\xi$ from 15 (late noise equal to early noise) to 100 (late noise much larger than early noise), we varied $\lambda$ from 0 (no leak) to 1 (maximum leak), and considered three different values of selective gating: high gating, $w=.35$, moderate gating, $w=.65$, and low gating, $w=.85$.

\footnotetext{
${ }^{10}$ The Matlab code for running this simulation is available at https://figshare.com/s/24c7cdb825ccf04b731f.
} 
Figure 1 displays the results of our simulation study. In all graphs we plot $P_{A B}$ under both F-SI and the SI model as a function of model parameter values. The rows correspond to gating conditions: high, moderate, and low. The left-hand column plots $P_{A B}$ as a function of $\lambda$, the right-hand column plots $P_{A B}$ as a function of $\xi$. The three graphs in the left-hand column were calculated under $\xi=\sigma=15$. The three graphs in the right-hand column were calculated under $\lambda=.5$.

Overall, we find that F-SI approximates the SI model very well when late noise, $\xi$, is larger than early noise, $\sigma$. This follows from the normally distributed random variable $\xi \cdot \boldsymbol{\zeta}_{A}(t)$ (resp. $\xi \cdot \boldsymbol{\zeta}_{B}(t)$ ) having proportionally greater influence on the accumulator random variable $\boldsymbol{Y}_{A}(t)$ (resp. $\left.\boldsymbol{Y}_{B}(t)\right)$ when $\xi$ is large relative to $\sigma$; this leaves the distribution of $\boldsymbol{Y}_{A}(t)$ (resp. $\boldsymbol{Y}_{B}(t)$ ) approximately normal in shape. We find that F-SI performs less well as an approximation to SI as selective gating increases ( $w$ decreases). When selective gating is high, the random variables $\boldsymbol{I}_{A}(t)$ and $\boldsymbol{I}_{B}(t)$ tend to be bimodal in distribution and are not well approximated by the normally distributed random variables, $\boldsymbol{I}_{A}^{\prime}(t)$ and $\boldsymbol{I}_{B}^{\prime}(t)$, despite having the same expectation and variances. While our simulation study suggests that F-SI is a good approximation of the SI model, it is limited in scope and is intended as an illustration.

\section{Conclusions and Discussion.}

We have made a series of theoretical connections between the SI model of Tsetsos et al. (2016a) and several classic models of probabilistic binary choice. In addition to providing analytical formulae for multiple versions of SI, we demonstrated the explicit connections between SI and the constraints on binary choice probabilities, weak stochastic transitivity and the triangle inequalities, that Tsetsos et al. evaluated statistically on their data. As an additional insight into SI, it follows from Equation 6 that early and late noise are mathematically indistinguishable when $w=1$. Therefore, late and early noise cannot be conceptually teased apart under that condition.

Our results apply to the selective integration theory of Tsetsos et al. (2016a) as described and implemented in their paper. Our results may not hold under more general applications, such as situations where evidence accumulation is terminated by a response boundary - see Ratcliff (2006) for an illustration of how evidence accumulation distributions can be altered in such cases.

Our characterizations, in the tradition of the axiomatic approach, keep track of auxiliary assumptions, notably distributional assumptions, that a more general version of Tsetsos et al.'s model could omit. Much of their approach is aimed at modeling violations of transitivity. While transitivity is an algebraic (non-probabilistic) axiom, Tsetsos et al.'s model is a stochastic process. The conceptual, mathematical, and 

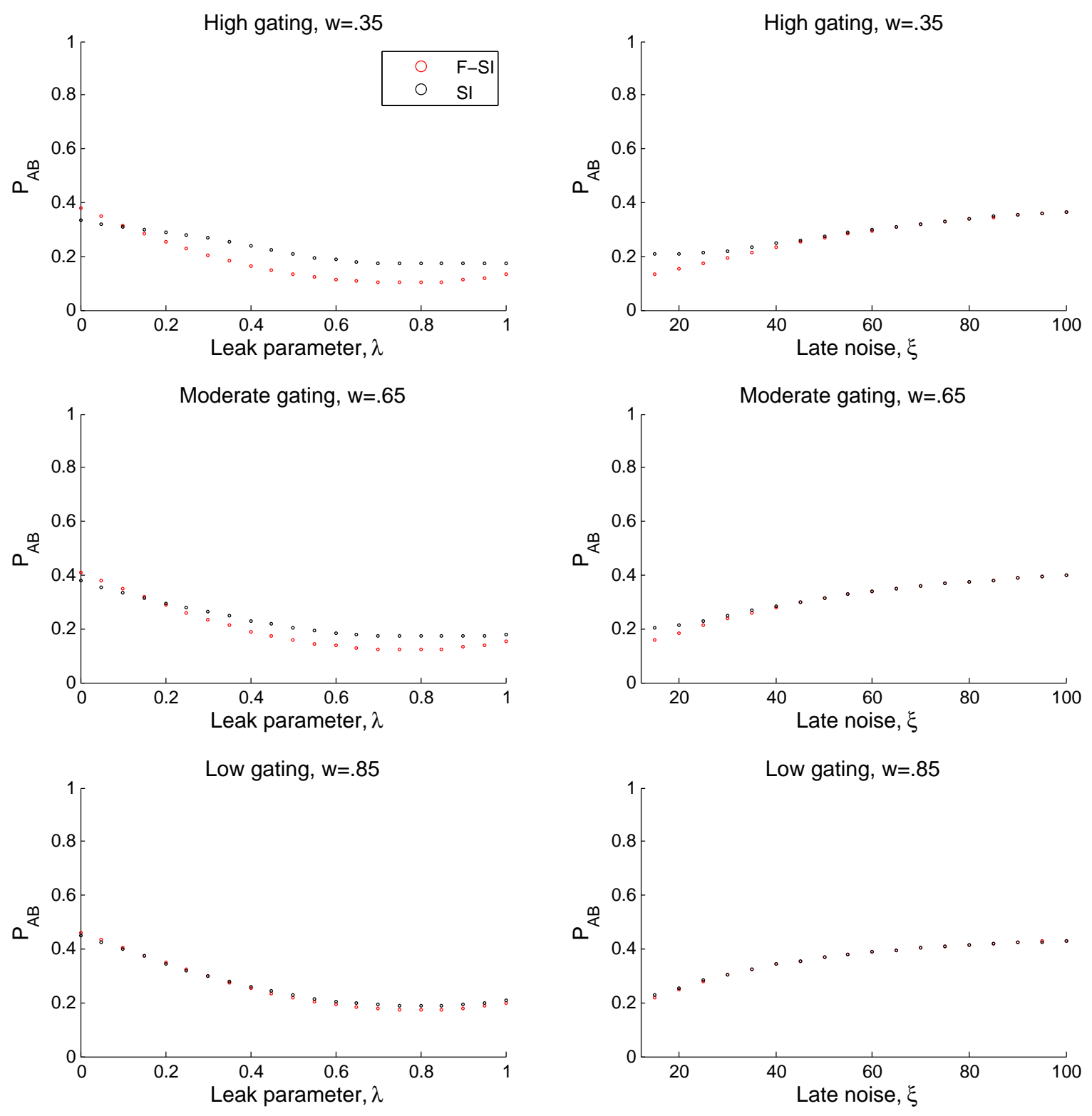

17

Figure 1: Each graph compares our Fechnerian model to the SI model by plotting $P_{A B}$ as functions of: $w, \lambda$, and $\xi$. In all graphs, $\sigma=15$. 
statistical gap between algebraic axioms and probabilistic data has been the focus of heavy research in the literature on axiom testing and on probabilistic choice models (see, e.g., Carbone and Hey, 2000, Falmagne, 1978, 1980, 1983; Falmagne and Doignon, 1997; Harless and Camerer, 1994; Hey, 1995, 2005, Hey and Orme, 1994; Iverson, 1990; Iverson and Falmagne, 1985, Loomes and Sugden, 1995; Luce, 1959, 1997; Luce and Narens, 1994; Luce and Suppes, 1965, Regenwetter, 1996, Regenwetter et al., 1999; Regenwetter and Marley, 2001). Building on this tradition, our approach sheds a conceptual and analytical light on the relationship between Tsetsos et al.'s model and the major types of probabilistic specifications of transitivity in the axiom testing literature.

ACKnowledgments. This work was supported by the National Science Foundation (SES-14-59866, PI: Davis-Stober \& SES-14-59699, PI: Regenwetter) and the National Institutes of Health (K25AA024182, PI: Davis-Stober). Any opinions, findings, and conclusions or recommendations expressed in this publication are those of the authors and do not necessarily reflect the views of the National Science Foundation, National Institutes of Health or of the authors' universities.

\section{Appendix}

Proof of Lemma 4. We calculate $E\left[\boldsymbol{I}_{A}(t)\right]$ and $E\left[\boldsymbol{I}_{B}(t)\right]$ by applying the law of total expectation,

$$
\begin{aligned}
& E\left[\boldsymbol{I}_{A}(t)\right]=E\left[\boldsymbol{I}_{A}(t) \mid \theta=1\right] \operatorname{Pr}(\theta=1)+E\left[\boldsymbol{I}_{A}(t) \mid \theta=w\right] \operatorname{Pr}(\theta=w), \\
& E\left[\boldsymbol{I}_{B}(t)\right]=E\left[\boldsymbol{I}_{B}(t) \mid \theta=1\right] \operatorname{Pr}(\theta=1)+E\left[\boldsymbol{I}_{B}(t) \mid \theta=w\right] \operatorname{Pr}(\theta=w) .
\end{aligned}
$$

These equations can be rewritten as

$$
\begin{aligned}
& E\left[\boldsymbol{I}_{A}(t)\right]=E\left[\boldsymbol{I}_{A}(t) \mid \theta=1\right] \operatorname{Pr}\left(\boldsymbol{X}_{A}(t)>\boldsymbol{X}_{B}(t)\right)+E\left[\boldsymbol{I}_{A}(t) \mid \theta=w\right] \operatorname{Pr}\left(\boldsymbol{X}_{B}(t)>\boldsymbol{X}_{A}(t)\right), \\
& E\left[\boldsymbol{I}_{B}(t)\right]=E\left[\boldsymbol{I}_{B}(t) \mid \theta=1\right] \operatorname{Pr}\left(\boldsymbol{X}_{B}(t)>\boldsymbol{X}_{A}(t)\right)+E\left[\boldsymbol{I}_{B}(t) \mid \theta=w\right] \operatorname{Pr}\left(\boldsymbol{X}_{A}(t)>\boldsymbol{X}_{B}(t)\right) .
\end{aligned}
$$

Calculating the conditional expectations yields

$$
\begin{gathered}
E\left[\boldsymbol{I}_{A}(t)\right]=\frac{\int_{-\infty}^{\infty} \int_{-\infty}^{x_{A}} x_{A} h\left(x_{A}, x_{B}\right) d x_{B} d x_{A}}{\int_{-\infty}^{\infty} \int_{-\infty}^{x_{A}} h\left(x_{A}, x_{B}\right) d x_{B} d x_{A}} \operatorname{Pr}\left(\boldsymbol{X}_{A}(t)>\boldsymbol{X}_{B}(t)\right)+ \\
\frac{w \int_{-\infty}^{\infty} \int_{x_{A}}^{\infty} x_{A} h\left(x_{A}, x_{B}\right) d x_{B} d x_{A}}{\int_{-\infty}^{\infty} \int_{x_{A}}^{\infty} h\left(x_{A}, x_{B}\right) d x_{B} d x_{A}} \operatorname{Pr}\left(\boldsymbol{X}_{B}(t)>\boldsymbol{X}_{A}(t)\right)
\end{gathered}
$$




$$
\begin{gathered}
E\left[\boldsymbol{I}_{B}(t)\right]=\frac{\int_{-\infty}^{\infty} \int_{-\infty}^{x_{B}} x_{B} h\left(x_{A}, x_{B}\right) d x_{A} d x_{B}}{\int_{-\infty}^{\infty} \int_{-\infty}^{x_{B}} h\left(x_{A}, x_{B}\right) d x_{A} d x_{B}} \operatorname{Pr}\left(\boldsymbol{X}_{B}(t)>\boldsymbol{X}_{A}(t)\right)+ \\
\frac{w \int_{-\infty}^{\infty} \int_{x_{B}}^{\infty} x_{B} h\left(x_{A}, x_{B}\right) d x_{A} d x_{B}}{\int_{-\infty}^{\infty} \int_{x_{B}}^{\infty} h\left(x_{A}, x_{B}\right) d x_{A} d x_{B}} \operatorname{Pr}\left(\boldsymbol{X}_{A}(t)>\boldsymbol{X}_{B}(t)\right) .
\end{gathered}
$$

Next, we observe that

$$
\begin{gathered}
\operatorname{Pr}\left(\boldsymbol{X}_{A}>\boldsymbol{X}_{B}\right)=\int_{-\infty}^{\infty} \int_{-\infty}^{x_{A}} h\left(x_{A}, x_{B}\right) d x_{B} d x_{A}=\int_{-\infty}^{\infty} \int_{x_{B}}^{\infty} h\left(x_{A}, x_{B}\right) d x_{A} d x_{B}, \\
=\phi\left(\frac{s_{A}(t)-s_{B}(t)}{\sqrt{2 \sigma^{2}}}\right),
\end{gathered}
$$

and

$$
\begin{gathered}
\operatorname{Pr}\left(\boldsymbol{X}_{B}>\boldsymbol{X}_{A}\right)=\int_{-\infty}^{\infty} \int_{x_{A}}^{\infty} h\left(x_{A}, x_{B}\right) d x_{B} d x_{A}=\int_{-\infty}^{\infty} \int_{-\infty}^{x_{B}} h\left(x_{A}, x_{B}\right) d x_{A} d x_{B}, \\
=\phi\left(\frac{s_{B}(t)-s_{A}(t)}{\sqrt{2 \sigma^{2}}}\right) .
\end{gathered}
$$

Finally, after simplifying, Equations 16 and 17 become

$$
\begin{aligned}
& E\left[\boldsymbol{I}_{A}(t)\right]=\int_{-\infty}^{\infty} \int_{-\infty}^{x_{A}} x_{A} h\left(x_{A}, x_{B}\right) d x_{B} d x_{A}+w \int_{-\infty}^{\infty} \int_{x_{A}}^{\infty} x_{A} h\left(x_{A}, x_{B}\right) d x_{B} d x_{A}, \\
& E\left[\boldsymbol{I}_{B}(t)\right]=\int_{-\infty}^{\infty} \int_{-\infty}^{x_{B}} x_{B} h\left(x_{A}, x_{B}\right) d x_{A} d x_{B}+w \int_{-\infty}^{\infty} \int_{x_{B}}^{\infty} x_{B} h\left(x_{A}, x_{B}\right) d x_{A} d x_{B},
\end{aligned}
$$

which completes the proof.

Proof of Lemma 5. We calculate $\operatorname{Var}\left[\boldsymbol{I}_{A}(t)\right]$ and $\operatorname{Var}\left[\boldsymbol{I}_{B}(t)\right]$ by applying the law of total variance,

$$
\begin{aligned}
& \operatorname{Var}\left[\boldsymbol{I}_{A}(t)\right]=E\left[\operatorname{Var}\left[\boldsymbol{I}_{A}(t) \mid \theta\right]+\operatorname{Var}\left[E\left[\boldsymbol{I}_{A}(t) \mid \theta\right]\right],\right. \\
& \operatorname{Var}\left[\boldsymbol{I}_{B}(t)\right]=E\left[\operatorname{Var}\left[\boldsymbol{I}_{B}(t) \mid \theta\right]+\operatorname{Var}\left[E\left[\boldsymbol{I}_{B}(t) \mid \theta\right]\right] .\right.
\end{aligned}
$$


These equations can be rewritten as

$$
\begin{aligned}
& \operatorname{Var}\left[\boldsymbol{I}_{A}(t)\right]=\operatorname{Var}\left[\boldsymbol{I}_{A}(t) \mid \theta=1\right] \operatorname{Pr}\left(\boldsymbol{X}_{A}(t)>\boldsymbol{X}_{B}(t)\right) \\
& \quad+\operatorname{Var}\left[\boldsymbol{I}_{A}(t) \mid \theta=w\right] \operatorname{Pr}\left(\boldsymbol{X}_{B}(t)>\boldsymbol{X}_{A}(t)\right) \\
& +\operatorname{Pr}\left(\boldsymbol{X}_{A}(t)>\boldsymbol{X}_{B}(t)\right)\left(E\left[\boldsymbol{I}_{A}(t) \mid \theta=1\right]-E\left[I_{A}(t)\right]\right)^{2} \\
& \quad+\operatorname{Pr}\left(\boldsymbol{X}_{B}(t)>\boldsymbol{X}_{A}(t)\right)\left(E\left[\boldsymbol{I}_{A}(t) \mid \theta=w\right]-E\left[I_{A}(t)\right]\right)^{2},
\end{aligned}
$$

$$
\begin{aligned}
\operatorname{Var}\left[\boldsymbol{I}_{B}(t)\right]=\operatorname{Var}\left[\boldsymbol{I}_{B}(t) \mid \theta=1\right] & \operatorname{Pr}\left(\boldsymbol{X}_{B}(t)>\boldsymbol{X}_{A}(t)\right) \\
& +\operatorname{Var}\left[\boldsymbol{I}_{B}(t) \mid \theta=w\right] \operatorname{Pr}\left(\boldsymbol{X}_{A}(t)>\boldsymbol{X}_{B}(t)\right) \\
+\operatorname{Pr}\left(\boldsymbol{X}_{B}(t)\right. & \left.>\boldsymbol{X}_{A}(t)\right)\left(E\left[\boldsymbol{I}_{B}(t) \mid \theta=1\right]-E\left[I_{B}(t)\right]\right)^{2} \\
& +\operatorname{Pr}\left(\boldsymbol{X}_{A}(t)>\boldsymbol{X}_{B}(t)\right)\left(E\left[\boldsymbol{I}_{B}(t) \mid \theta=w\right]-E\left[I_{B}(t)\right]\right)^{2} .
\end{aligned}
$$

Calculating the conditional expectations and simplifying yields

$$
\begin{aligned}
& \operatorname{Var}\left[\boldsymbol{I}_{A}(t)\right]=\int_{-\infty}^{\infty} \int_{-\infty}^{x_{A}} x_{A}^{2} h\left(x_{A}, x_{B}\right) d x_{B} d x_{A}-\frac{\left(\int_{-\infty}^{\infty} \int_{-\infty}^{x_{A}} x_{A} h\left(x_{A}, x_{B}\right) d x_{B} d x_{A}\right)^{2}}{\phi\left(\frac{s_{A}(t)-s_{B}(t)}{\sqrt{2 \sigma^{2}}}\right)} \\
&+ w^{2} \int_{-\infty}^{\infty} \int_{x_{A}}^{\infty} x_{A}^{2} h\left(x_{A}, x_{B}\right) d x_{B} d x_{A}-\frac{\left(w \int_{-\infty}^{\infty} \int_{x_{A}}^{\infty} x_{A} h\left(x_{A}, x_{B}\right) d x_{B} d x_{A}\right)^{2}}{\phi\left(\frac{s_{B}(t)-s_{A}(t)}{\sqrt{2 \sigma^{2}}}\right)} \\
&+\phi\left(\frac{s_{A}(t)-s_{B}(t)}{\sqrt{2 \sigma^{2}}}\right)\left(\frac{\int_{-\infty}^{\infty} \int_{-\infty}^{x_{A}} x_{A} h\left(x_{A}, x_{B}\right) d x_{B} d x_{A}}{\phi\left(\frac{s_{A}(t)-s_{B}(t)}{\sqrt{2 \sigma^{2}}}\right)}-E\left[I_{A}(t)\right]\right)^{2} \\
&+\phi\left(\frac{s_{B}(t)-s_{A}(t)}{\sqrt{2 \sigma^{2}}}\right)\left(\frac{w \int_{-\infty}^{\infty} \int_{x_{A}}^{\infty} x_{A} h\left(x_{A}, x_{B}\right) d x_{B} d x_{A}}{\phi\left(\frac{s_{B}(t)-s_{A}(t)}{\sqrt{2 \sigma^{2}}}\right)}-E\left[I_{A}(t)\right]\right)^{2},
\end{aligned}
$$




$$
\begin{aligned}
& \operatorname{Var}\left[\boldsymbol{I}_{B}(t)\right]=\int_{-\infty}^{\infty} \int_{-\infty}^{x_{B}} x_{B}^{2} h\left(x_{A}, x_{B}\right) d x_{A} d x_{B}-\frac{\left(\int_{-\infty}^{\infty} \int_{-\infty}^{x_{B}} x_{B} h\left(x_{A}, x_{B}\right) d x_{A} d x_{B}\right)^{2}}{\phi\left(\frac{s_{B}(t)-s_{A}(t)}{\sqrt{2 \sigma^{2}}}\right)} \\
&+ w^{2} \int_{-\infty}^{\infty} \int_{x_{B}}^{\infty} x_{B}^{2} h\left(x_{A}, x_{B}\right) d x_{A} d x_{B}-\frac{\left(w \int_{-\infty}^{\infty} \int_{x_{B}}^{\infty} x_{B} h\left(x_{A}, x_{B}\right) d x_{A} d x_{B}\right)^{2}}{\phi\left(\frac{s_{A}(t)-s_{B}(t)}{\sqrt{2 \sigma^{2}}}\right)} \\
&+\phi\left(\frac{s_{B}(t)-s_{A}(t)}{\sqrt{2 \sigma^{2}}}\right)\left(\frac{\int_{-\infty}^{\infty} \int_{-\infty}^{x_{B}} x_{B} h\left(x_{A}, x_{B}\right) d x_{A} d x_{B}}{\phi\left(\frac{s_{B}(t)-s_{A}(t)}{\sqrt{2 \sigma^{2}}}\right)}-E\left[I_{B}(t)\right]\right)^{2} \\
&+\phi\left(\frac{s_{A}(t)-s_{B}(t)}{\sqrt{2 \sigma^{2}}}\right)\left(\frac{w \int_{-\infty}^{\infty} \int_{x_{B}}^{\infty} x_{B} h\left(x_{A}, x_{B}\right) d x_{A} d x_{B}}{\phi\left(\frac{s_{A}(t)-s_{B}(t)}{\sqrt{2 \sigma^{2}}}\right)}-E\left[I_{B}(t)\right]\right)^{2},
\end{aligned}
$$

which completes the proof. 
Birnbaum, M., Beeghley, D., 1997. Violations of branch independence in judgments of the value of gambles. Psychological Science 8, 87-94.

Birnbaum, M. H., 2005. Three new tests of independence that differentiate models of risky decision making. Management Science 51, 1346-1358.

Block, H. D., Marschak, J., 1960. Random orderings and stochastic theories of responses. In: Olkin, I., Ghurye, S., Hoeffding, H., Madow, W., Mann, H. (Eds.), Contributions to Probability and Statistics. Stanford University Press, Stanford, pp. $97-132$.

Brown, S., Heathcote, A., 2005. A ballistic model of choice response time. Psychological Review 112 (117-128).

Brown, S. D., Heathcote, A., 2008. The simplest complete model of choice response time: Linear ballistic accumulation. Cognitive Psycholog 57 (153-178).

Busemeyer, J. R., Townsend, J. T., 1993. Decision field theory: A dynamic-cognitive approach to decision making in an uncertain environment. Psychological Review $100,432-459$.

Carbone, E., Hey, J. D., 2000. Which error story is best? Journal of Risk and Uncertainty 20, 161-176.

Cohen, M., Falmagne, J.-C., 1978. Random scale representations of binary choice probabilities: A counterexample to a conjecture of Marschak., unpublished manuscript, Department of Psychology, New York University, NY.

Dagsvik, J., 2008. Axiomatization of stochastic models for choice under uncertainty. Mathematical Social Sciences 55, 341-370.

Davis-Stober, C., 2012. A lexicographic semiorder polytope and probabilistic representations of choice. Journal of Mathematical Psychology 56, 86-94.

Davis-Stober, C., Brown, N., 2013. Evaluating decision making "type" under padditive utility representations. Journal of Mathematical Psychology 57, 320-328.

Davis-Stober, C. P., 2009. Analysis of multinomial models under inequality constraints: Applications to measurement theory. Journal of Mathematical Psychology $53,1-13$. 
Davis-Stober, C. P., Park, S., Brown, N., Regenwetter, M., 2016. Reported violations of rationality may be aggregation artifacts. Proceedings of the National Academy of Sciences of the United States of America. Online at www.pnas.org/cgi/doi/10.1073/pnas.1606997113.

Falmagne, J.-C., 1978. A representation theorem for finite random scale systems. Journal of Mathematical Psychology 18, 52-72.

Falmagne, J.-C., 1980. A probabilistic theory of extensive measurement. Philosophy of Science 47, 277-296.

Falmagne, J.-C., 1983. A random utility model for a belief function. Synthèse 57, $35-48$.

Falmagne, J.-C., Doignon, J.-P., 1997. Stochastic evolution of rationality. Theory and Decision 43, 107-138.

Harless, D. W., Camerer, C. F., 1994. The predictive value of generalized expected utility theories. Econometrica 62, 1251-1289.

Hey, J. D., 1995. Experimental investigations of errors in decision making under risk. European Economic Review 39, 633-640.

Hey, J. D., 2005. Why we should not be silent about noise. Experimental Economics $8,325-345$.

Hey, J. D., Orme, C., 1994. Investigating generalizations of expected utility theory using experimental data. Econometrica 62, 1291-1326.

Iverson, G. J., 1990. Probabilistic measurement theory, available as MBS 90-23 Technical Report at the IMBS, University of California, Irvine.

Iverson, G. J., Falmagne, J.-C., 1985. Statistical issues in measurement. Mathematical Social Sciences 10, 131-153.

Karabatsos, G., 2005. The exchangeable multinomial model as an approach to testing deterministic axioms of choice and measurement. Journal of Mathematical Psychology 49, $51-69$.

Krantz, D. H., Luce, R. D., Suppes, P., Tversky, A., 1971. Foundations of Measurement. Vol. 1. Academic Press, San Diego. 
Loomes, G., Sugden, R., 1982. Regret theory: An alternative theory of rational choice under uncertainty. Economic Journal 92, 805-824.

Loomes, G., Sugden, R., 1995. Incorporating a stochastic element into decision theories. European Economic Review 39, 641-648.

Luce, R. D., 1956. Semiorders and a theory of utility discrimination. Econometrica $24,178-191$.

Luce, R. D., 1959. Individual Choice Behavior: A Theoretical Analysis. John Wiley, New York.

Luce, R. D., 1977. The choice axiom after twenty years. Journal of Mathematical Psychology 15, 215-233.

Luce, R. D., 1997. Several unresolved conceptual problems of mathematical psychology. Journal of Mathematical Psychology 41, 79-87.

Luce, R. D., 2000. Utility of Gains and Losses Measurement-Theoretical and Experimental Approaches. Lawrence Erlbaum Associates, Mahwah, New Jersey.

Luce, R. D., Krantz, D. H., Suppes, P., Tversky, A., 1990. Foundations of Measurement. Vol. 3. Academic Press, San Diego.

Luce, R. D., Marley, A. A. J., 2005. Ranked additive utility representations of gambles: Old and new axiomatizations. Journal of Risk and Uncertainty 30, 21-62.

Luce, R. D., Narens, L., 1994. Fifteen problems in the representational theory of measurement. In: Humphreys, P. (Ed.), Patrick Suppes: Scientific Philosopher, volume 2: Philosophy of Physics, Theory Structure, Measurement Theory, Philosophy of Language, and Logic. Kluwer, Dordrecht, pp. 219-245.

Luce, R. D., Suppes, P., 1965. Preference, utility and subjective probability. In: Luce, R. D., Bush, R. R., Galanter, E. (Eds.), Handbook of Mathematical Psychology. Vol. III. Wiley, New York, pp. 249-410.

Marley, A. A. J., 2002. A simple axiomatization of binary rank-dependent utility of gains (losses). Journal of Mathematical Psychology 46, 40-55.

McFadden, D., 1973. Conditional logit analysis of qualitative choice behavior. In: Zarembka, P. (Ed.), Frontiers in Econometrics. Academic Press, New York. 
Müller-Trede, J., Sher, S., McKenzie, C. R., 2015. Transitivity in context: A rational analysis of intransitive choice and context-sensitive preference. Decision 2 (4), 280305.

Myung, J., Karabatsos, G., Iverson, G., 2005. A Bayesian approach to testing decision making axioms. Journal of Mathematical Psychology 49, 205-225.

Narens, L., 1985. Abstract Measurement Theory. MIT Press, Cambridge, MA.

Otter, T., Allenby, G., van Zandt, T., 2008. An integrated model of discrete choice and response time. Journal of Marketing Research 45, 593-607.

Ratcliff, R., 2006. Modeling response signal and response time data. Cognitive psychology 53 (3), 195-237.

Ratcliff, R., Rouder, J., 1998. Modeling response times for two-choice decisions. Psychological Science 9, 347-356.

Ratcliff, R., Starns, J., 2009. Modeling confidence and response time in recognition memory. Psychological Review 116, 59-83.

Regenwetter, M., 1996. Random utility representations of finite m-ary relations. Journal of Mathematical Psychology 40, 219-234.

Regenwetter, M., Dana, J., Davis-Stober, C. P., 2010. Testing transitivity of preferences on two-alternative forced choice data. Frontiers in Quantitative Psychology and Measurement.

Regenwetter, M., Dana, J., Davis-Stober, C. P., 2011. Transitivity of preferences. Psychological Review 118, 42-56.

Regenwetter, M., Davis-Stober, C. P., 2012. Behavioral variability of choices versus structural inconsistency of preferences. Psychological Review 119 (2), 408-416.

Regenwetter, M., Davis-Stober, C. P., Lim, S. H., Guo, Y., Popova, A., Zwilling, C., Cha, Y.-C., Messner, W., 2014. QTEST: quantitative testing of theories of binary choice. Decision 1 (1), 2-34.

Regenwetter, M., Falmagne, J.-C., Grofman, B., 1999. A stochastic model of preference change and its application to 1992 presidential election panel data. Psychological Review 106, 362-384. 
Regenwetter, M., Marley, A. A. J., 2001. Random relations, random utilities, and random functions. Journal of Mathematical Psychology 45, 864-912.

Regenwetter, M., Müller-Trede, J., 2016. Random utility without regularity, manuscript.

Roberts, F. S., 1979. Measurement Theory. Addison-Wesley, London.

Roe, R. M., Busemeyer, J. R., Townsend, J. T., 2001. Multialternative decision field theory: A dynamic connectionist model of decision making. Psychological Review 108, 370-392.

Scott, D., Suppes, P., 1958. Foundational aspects of theories of measurement. Journal of Symbolic Logic 23, 113-128.

Smith, P. L., Ratcliff, R., 2009. An integrated theory of attention and decision making in visual signal detection. Psychological review 116 (2), 283.

Suppes, P., Krantz, D. H., Luce, R. D., Tversky, A., 1989. Foundations of Measurement. Vol. II. Academic Press, San Diego.

Suppes, P., Zanotti, M., 1992. Qualitative axioms for a random-variable representation of extensive quantities. In: Savage, C. W., Ehrlich, P. (Eds.), Philosophical and Foundational Issues in Measurement Theory. Laurence Erlbaum, Hillsdale, NJ, pp. 40-52.

Thurstone, L. L., 1927. A law of comparative judgement. Psychological Review 34, $273-286$.

Townsend, J., Houpt, J., Silbert, N., 2012. General recognition theory extended to include response times: Predictions for a class of parallel systems. Journal of Mathematical Psychology 56, 476-494.

Trueblood, J., Brown, S., Heathcote, A., Busemeyer, J., 2014. The multi-attribute linear ballistic accumulator model. Psychological Review 121, 179-205.

Tsetsos, K., Moran, R., Moreland, J., Chater, N., Usher, M., Summerfield, C., 2016a. Economic irrationality is optimal during noisy decision making. Proceedings of the National Academy of Sciences of the United States of America 113 (1), 3102-7. 
Tsetsos, K., Moran, R., Moreland, J. C., Chater, N., Usher, M., Summerfield, C., 2016b. Reply to davis-stober et al.: Violations of rationality in a psychophysical task are not aggregation artifacts. Proceedings of the National Academy of Sciences, 201608989.

Tversky, A., 1969. Intransitivity of preferences. Psychological Review 76, 31-48.

Wagenmakers, E., van der Maas, H., Grasman, R., 2007. An ez-diffusion model for response time and accuracy. Psychonomic Bulletin \& Review 14, 3-22.

Wakker, P. P., Tversky, A., 1993. An axiomatization of cumulative prospect theory. Journal of Risk and Uncertainty 7, 147-176.

Yellott, J. I. J., 1977. The relationship between Luce's choice axiom, Thurstone's theory of comparative judgement, and the double exponential distribution. Journal of Mathematical Psychology 15, 109-144. 